All letters are subject to editing and may be shortened. Letters should be sent to the BJGP office by e-mail in the first instance, addressed to

journal@rcgp.org.uk (please include your postal address). Alternatively, they may be sent by post as an MS Word or plain text version on CD or DVD. We regret that we cannot notify authors regarding publication. Letters not published in the Journal may be posted online on our Discussion Forum. For instructions please visit: http://www.rcgp.org.uk/bjgp-discuss

\section{Hypertension in Pakistan}

I am concerned about this long letter. ${ }^{1}$ I find it odd and I do not understand why it was accepted for publication. There are two reasons for this concern. Firstly, I find it simplistic in its acceptance of the usefulness of screening for a single risk factor (hypertension) and secondly, I am suspicious of the authors' motives in writing.

I have lived and worked for a number of years in Pakistan, and have ongoing connections with the country. Part of my role was in diabetes management, but I very rapidly became disillusioned with regard to treatment recommendations that are based on a developed country model. On my desktop I have, with the permission of Cambridge University (http://www.dtu.ox.ac.uk/Outcomesmode I), the UKPDS Outcomes Model programme that was acquired in order to do some research to prove that which I already know. We all do really. That is, for the vast majority of the population, the recommendation to buy expensive pharmaceutical preparations over many years in order to, largely theoretically, save a few months of life or morbidity, does not take into consideration informed consent.

The true cost of implementation of this recommendation is to deprive individuals and families of essentials such as food, shelter, and schooling. Advice from professionals to act in this way is often treated with a respect that is simply not deserved and this when there is no axe to grind. This is true in nations that are considered developed, and even more so in countries that are less fortunate and for which the profit motive in selling pharmaceuticals direct to the public is much less hidden.

With regard to my other concern, I was interested to see that the address of your correspondents is the department of Pharmaceutical Sciences in a university in Malaysia. This is at least honest, but it does not reassure me about their objectivity. Intriguingly, Balochistan is one of the least developed areas of Pakistan and in this context I really cannot see how the practical application of their ideas can be remotely achieved.

\section{Jim Newmark,}

Salaried GP for Asylum Seekers, Refugees, and Homeless, Bevan House Primary Care Centre, Bradford, BD1 2LT.

E-mail: jim.newmark@bradford.nhs.uk

\section{REFERENCE}

1. Saleem F, Hassali AA, Shafie AA. Hypertension in Pakistan: time to take some serious action. Br J Gen Pract 2010; 60(575): 449-450.

DOI: 10.3399/bjgp10X515142

\section{Authors' response}

We truly appreciate the response by $\mathrm{Dr}$ Newmark about our recent letter and would like to clarify a few points raised by him. First, he expressed his concerns about our credibility and authority to suggest recommendations for healthcare issues in Pakistan, based on our affiliation with a Malaysian university. For all of our readers' information, the first author of our letter is a qualified practising clinical pharmacist from Pakistan and currently is affiliated with the Discipline of Social and Administrative Pharmacy, School of Pharmaceutical Sciences at Universiti Sains Malaysia for his PhD studies. As a pharmacist in the ground work, Mr Fahad is well versed with the situation in Pakistan to a great extent, especially to the region in which he is currently practising (Balochistan).

Second, Dr Newmark has also argued that our recommendations are merely based on western treatment guidelines and are not suitable for application in Pakistan. Based on the authors' current observation of recent practice in Pakistan, we are afraid that he missed the point that things had changed positively over the last few years. There are now adoptions of a number of standard international guidelines in practice, such as the Joint National Committee, British Society of Hypertension Management, European Society of Cardiology, and Canadian Hypertension Education Program, the employment of more foreign trained doctors, and an increasing awareness of evidence-based practice by practitioners and national health authorities.

The initial letter that we wrote was to highlight the issues related to nonadherence and poor knowledge towards hypertension and, as practising pharmacists, to develop or at least discuss a mechanism to improve the condition. The word 'pharmacist' is relatively new to the people, and the profession is still struggling to be recognised by the healthcare team and institution. Therefore, it was stressed in the letter that interprofessional roles have to be strengthened and the pharmacist must work out of traditional domains of dispensing and supply, and start looking ahead for further responsibilities in patient care.

The letter had nothing to do with the treatment guidelines, the manner of how hypertension is treated, or to discuss singular versus multiple-risk factors. It was an effort to promote the role of 
pharmacists that will be beneficial for all. We are quite sure that if the new system is implemented and the pharmacist is ready to be moulded into a new shape, practical application of these ideas can be achieved even in the least developed areas of Pakistan. It's better to try than to sit and wait for a miracle to happen.

\section{Fahad Saleem,}

B-Pharm, MPhil, MBA(HRM), PhD candidate, Discipline of Social and Administrative Pharmacy, School of Pharmaceutical Sciences, Universiti Sains Malaysia. E-mail: fahaduob@gmail.com

\section{Mohamed Azmi Ahmad Hassali,} Senior Lecturer/HOD, Discipline of Social and Administrative Pharmacy, School of Pharmaceutical Sciences, Universiti Sains Malaysia.

\section{Asrul Akmal Shafie}

Senior Lecturer, Discipline of Social and Administrative Pharmacy, School of Pharmaceutical Sciences, Universiti Sains Malaysia.

\section{REFERENCE}

1. Saleem F, Hassali AA, Shafie AA. Hypertension in Pakistan: time to take some serious action. Br J Gen Pract 2010; 60(575): 449-550.

DOI: 10.3399/bjgp10X515151

\section{Access to computed tomography}

Thomas et al's study into primary care access to computed tomography (CT) scanning ${ }^{1}$ (that found that the provision of open access CT for chronic headache reduced referrals to neurology by $88 \%$ in the scanned group) is a helpful addition to a sparsely researched area. Its premise and conclusion, however, ought not to be accepted without further consideration. The premise, that it is desirable to avoid referral to neurology, seems questionable as the aim must surely be to reach a diagnosis leading to treatment and, therefore, symptomatic improvement. A scan rarely did that. Of the 30 patients that had scans and were also referred to neurologists, 13 (43\%) had a consultation that lead to the diagnosis of a treatable condition, mainly migraine.

A scan may have stopped $88 \%$ of referrals to neurology but did the patients feel better? CT in patients without clinical indicators may have provided short-term relief for doctor and patient, but in the absence of definite neurological indications the study demonstrates the risk: $10.2 \%$ had incidental abnormalities and in $5 \%$ the scan triggered the referral. Greater CT availability would surely lead to greater usage, more incidental abnormalities, and more referrals; while the average GP requested fewer than two CT scans, one GP requested seven.

GPs may refer fewer chronic headaches if open access CT is available. However, our patients would benefit more from an informed and skilled consultation (whether in primary or secondary care) leading to diagnosis and treatment, than from a scan leading to incidental findings or, more commonly, nothing.

\section{Nick Hall,}

Department of Neurology, Gloucester Royal Hospital, Gloucester, GL1 3NN.

E-mail: longhallage@googlemail.com

\section{REFERENCE}

1. Thomas R, Cook A, Main G, et al. Primary care access to computed tomography for chronic headache. $\mathrm{Br} J$ Gen Pract 2010; 60(575): 426-429.

DOI: 10.3399/bjgp10X515160

\section{Detection of congenital heart disease in mid-Essex}

Congenital heart defects account for $3 \%$ of all infant deaths, with $18-25 \%$ of affected infants dying in the first year of life. Early (pre-symptomatic) identification helps improve outcome. ${ }^{1}$ Routine prenatal anomaly scans detect less than half of cardiac defects, ${ }^{2,3}$ hence detection after birth remains important. Only families with a high risk of congenital heart defects are offered foetal echocardiography.

The UK National Screening Committee's newborn screening policy comprises clinical examination at birth and at 6-8 weeks, with specific cardiac investigations for high-risk children. ${ }^{4}$

We conducted a 3-year retrospective study in mid-Essex evaluating the detection of congenital heart defects, during postnatal and 6-8 week examinations from September 2004 to August 2007 on 55 infants born in midEssex with a confirmed diagnosis of congenital heart defects. Twenty infants identified either antenatally or diagnosed while admitted in the neonatal unit for other indications or detected by active case finding because comorbid conditions were excluded.

Results showed an incidence of 5.5/1000 live-births/year. Postnatal examination detected sixteen (45\%) and GPs detected 13 infants (37\%) during the 6-8 week check. Six infants with significant congenital heart defects were missed on screening and presented later with symptoms. Cyanotic lesions accounted for just under a quarter of all cases $(23.6 \%)$. The most common acyanotic lesion was ventricular septal defect (29\%). Six infants with significant cardiac lesions were missed on screening and presented at varying ages from

9 days to 7 months of whom four presented with cyanosis. Although all these infants survived, the increased morbidity, use of emergency care resources, and parental stress could be avoided by early detection.

Newborn physical examination is increasingly done in the first 24-48 hours of life by doctors in training and midwives. A congenital heart defect is likely to be missed because signs and symptoms are uncommon in the first few days, infants may look well initially, cyanosis is difficult to detect, and femoral pulses may be felt even in left ventricular obstructive lesions as a result of shunting. 